\title{
... Von der Qualitätssicherung der Qualitätssicherer der Qualität ...
}

\section{Christoph Bosshard}

Dr. med., Vizepräsident der FMH, Departementsverantwortlicher Daten, Demographie und Qualität / Schweizerische Akademie für Qualität in der Medizin SAQM

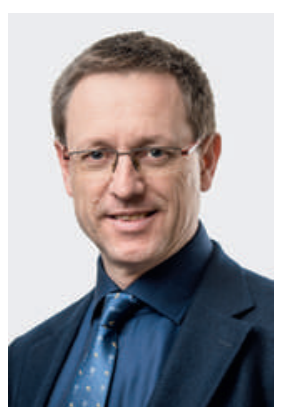

Mag der Quervergleich zum Titel von Dürrenmatts Novelle Der Auftrag oder Vom Beobachten des Beobachters der Beobachter etwas skurril erscheinen, so komme ich nicht umhin, etwas schmunzelnd daran zu denken, wenn ich nun diese einleitenden Zeilen zur Evaluation unserer SAQM schreibe.

Zum zweiten Geburtstag schenkten wir uns eine Evaluation. «Etwas sehr sportlich», bekamen wir von diverser Seite zu hören. Dennoch haben wir uns der Herausforderung bewusst gestellt: Wir wollten Fehlansätze frühzeitig erkennen und korrigieren können - ganz im Sinne eines lernenden Systems.

Nach mehreren Interviews, Befragungen und Gesprächen liegen nun die Resultate vor und finden Eingang in die Überarbeitung unserer Grundlagen und Stossrichtungen. Ich danke an dieser Stelle allen, welche uns ihr ehrliches Feedback gegeben haben. Wie heisst es so schön: Ihr Lob freut uns, Ihre Kritik bringt uns weiter!

Wir wollen Fehlansätze frühzeitig erkennen und korrigieren können - ganz im Sinne eines lernenden Systems.

Was wir aber unbedingt brauchen, ist Ihre Mitarbeit! Sei es als Vertreter einer der in der Ärztekammer vertretenen Organisationen im Forum Qualität, sei es als Partnerin und Partner ausserhalb der FMH im Rahmen der Einbindung im Dialog Qualität, sei es im Einbringen von Expertenwissen auf allen Ebenen und vor allem: als Ärztin und als Arzt in Ihrer alltäglichen Arbeit. Unser Bestreben, Sie hierin zu unterstützen, ist weder eine Einbahnstrasse, noch soll dieser Support von einer Flut von Formularen und Regulierungen geprägt sein.
Die erhaltenen Rückmeldungen sind allesamt spannend und werden für unsere weitere Arbeit berücksichtigt. Auch wenn diese Evaluation nun abgeschlossen ist und schon bald die dritte Kerze auf dem Geburtstagskuchen der SAQM brennen darf, so sind wir doch stets daran interessiert, von Ihnen zu hören, was Sie denken und was Sie sich wünschen. Ein strukturierter Prozess über die Basisorganisationen und Fachgesellschaften ist der Weg, der auch diese Institutionen unterstützt. Wir sind aber ebenso stets direkt erreichbar. Unsere Koordinaten finden Sie auf unserer Website www.saqm.ch.

\section{Die SAQM ist nicht Selbstzweck, sondern} das Instrument der FMH, um die Qualitätsaktivitäten ihrer Mitglieder-Organisationen transparent zu machen

Nun geht es also weiter. Die SAQM ist nicht Selbstzweck, sondern sie ist das Instrument der FMH, um die Qualitätsaktivitäten ihrer Mitglieder-Organisationen im Sinne der Effizienz zu koordinieren und transparent zu machen. Diese Transparenz benötigt in einer ersten Phase Vertraulichkeit zwecks Austauschs unter den Organisationen. Nach Erreichen des notwendigen Reifegrades und mit Zustimmung der Beteiligten wird die Transparenz gegen aussen hergestellt. $\mathrm{Da}$ in der FMH die Basis zuoberst steht, sind es auch Sie, sehr verehrte Kolleginnen und Kollegen, welche die SAQM ausmachen. Helfen Sie auch weiterhin mit, Teil dieser Erfolgsgeschichte zu sein. Ich danke Ihnen herzlich dafür. 